\section{Orale Antikoagulation nach Hirnblutung: Wann ist der richtige Zeitpunkt gekommen?}

\author{
Der Neubeginn einer oralen Antikoagulation bei Patienten mit Vorhof- \\ flimmern nach stattgehabter intrazerebraler Blutung ist eine Heraus- \\ forderung für die behandelnden Ärzte. Hierzu gibt es nun neue Erkenntnisse \\ aus einer schwedischen Beobachtungsstudie.
}

$\mathrm{D}$ ie Autoren analysierten retrospektiv Daten aus dem Riksstroke-Register, an dem in Schweden sämtliche Kliniken, die Patienten mit akutem Schlaganfall behandeln, teilnehmen, sowie Informationen aus Datenbanken, u. a. über Arzneimittelverordnungen und dem schwedischen Todesursachenregister.

Von Juli 2005 bis Dezember 2012 wurden in dieser Studie alle Patienten erfasst, die eine erstmalige nicht traumatische intrazerebrale Blutung erlitten hatten und bei denen gleichzeitig ein Vorhofflimmern vorlag. 2.619 Patienten wurden bis zu sieben Jahre nachbeobachtet. Die Studienpopulation war im Mittel 78 Jahre alt und überwiegend männlich (59,3\%). Der $\mathrm{CHA}_{2} \mathrm{DS}_{2}$-VAScScore lag bei $61 \%$ der Beteiligten zwischen 0 und 4, bei $39 \%$ bei 5 und höher.

Als primäre Endpunkte wurden zum einen thrombotische Ereignisse (ischämischer Schlaganfall, tödlicher Myokardinfarkt/systemische Embolie) und zum anderen hämorrhagische Ereignisse (erneute intrazerebrale Blutung und

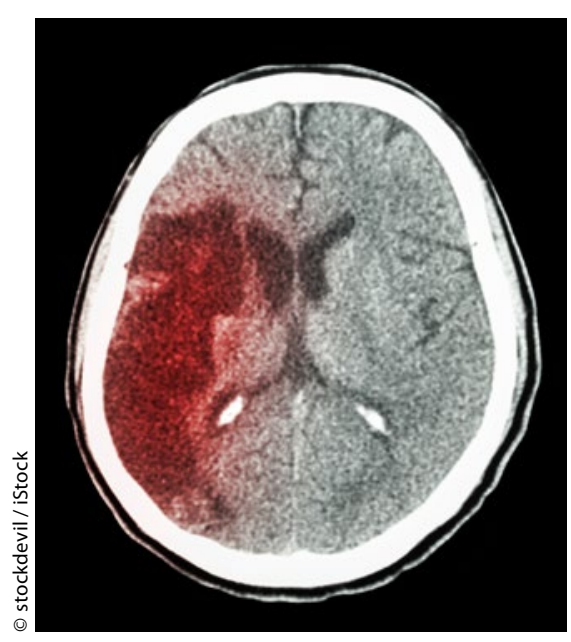

Nach einer intrazerebralen Blutung stellt sich die Frage, ob und wann erneut eine Antikoagulation erfolgen sollte. jede andere tödliche Blutung) definiert. Zur Analyse wurden die Patienten in drei Subgruppen je nach gerinnungswirksamer Medikation nach dem Indexereignis (keine Medikation: 1.251, orale Antikoagulation: 232, Plättchenhemmer: 1.136) unterteilt.

Während der Nachbeobachtung kam es zu 379 thrombotischen Ereignissen (davon $80 \%$ ischämische Schlaganfälle) und 96 hämorrhagischen Ereignissen (davon $83,5 \%$ erneute intrazerebrale Blutung). Das kumulative 3-Jahres-Risiko für ein thrombotisches Ereignis lag bei den Antikoagulierten bei 6,3\%, bei den Patienten ohne Medikation bei $13,8 \%$ und bei den Patienten unter Thrombozytenfunktionshemmern bei $18,8 \%$ (!). Die korrespondierenden Raten für Blutungsereignisse lagen bei 6,9, 4,4 und $3,9 \%$.

Somit erlitten Patienten mit oraler Antikoagulation nach einer intrazerebralen Blutung in den drei Folgejahren signifikant weniger thrombotische Ereignisse ohne signifikant erhöhte Blutungsgefahr. Dieses Ergebnis war konsistent für Männer und Frauen sowie auch für Patienten mit hohem Risiko $\left(\mathrm{CHA}_{2} \mathrm{DS}_{2}\right.$ VASc-Score $\geq 5$ ) und niedrigem Risiko. Als optimaler Zeitpunkt für den Wiederbeginn der oralen Antikoagulation ließ sich durch komplexe statistische Auswertungen der Zeitraum sieben bis acht Wochen nach dem Indexereignis identifizieren.

Pennlert J et al. Optimal Timing of Anticoagulant Treatment After Intracerebral Hemorrhage in Patients With Atrial Fibrillation. Stroke. 2017;48(2):314-20

\section{Kommentar}

Die orale Antikoagulation in der Primär- und Sekundärprävention ischämischer Schlaganfälle bei Patienten mit nicht valvulärem Vorhofflimmern ist heute etablierter Thera- piestandard. Kommt es hierunter jedoch zu einer intrazerebralen Blutung, stellt sich die Frage, ob ein Wiederbeginn indiziert ist und wenn ja, wann damit begonnen werden soll. Bis dato gibt es hierzu keine prospektiv erhobenen Daten. Daher stützen sich die Empfehlungen aus den Leitlinien (siehe u.a. aktuelle ESC-Leitlinie) auf Erkenntnisse aus Beobachtungsstudien. Hierbei mehren sich die Hinweise, dass Patienten nach einer intrazerebralen Blutung von einer Wiederaufnahme der Antikoagulation profitieren, insbesondere dann, wenn die Ursachen einer intrazerebralen Blutung zumindest teilweise kontrolliert werden können (z. B. optimierte Blutdruckeinstellung, Vermeidung einer Überdosierung der oralen Antikoagulation). Als optimaler Zeitpunkt scheint sich hier ein Zeitraum um acht Wochen nach dem Blutungsereignis herauszukristallisieren.

Dabei müssen jedoch auch in der hier zitierten Studie die Vielzahl möglicher Störfaktoren in retrospektiven Beobachtungsstudien berücksichtigt werden. $\mathrm{Zu}$ nennen sind insbesondere die niedrige Zahl der erneut antikoagulierten Patienten (hier 8,9\%), die fehlende Kontrolle von Verschreibungsfortsetzungen, Präparatwechsel und Therapieadhärenz des einzelnen Patienten, variabler Behandlungsbeginn, Indikationsentscheidung u.v.m. Aufgrund des Auswertzeitraums (bis Ende 2012) sind in der Arbeit die neuen/direkten oralen Antikoagulanzien noch nicht erfasst.

Inzwischen wurden prospektive Studien zur Behandlung von Patienten mit Vorhofflimmern nach intrazerebraler Blutung begonnen, u. a. APACHE-AF, in der Apixaban gegen Thrombozytenfunktionshemmer bzw. gar keine Medikation getestet wird und STROKECLOSE, in der der Verschluss des linken Vorhofohrs gegen verschiedene medikamentöse Therapiestrategien untersucht wird. Bis diese Ergebnisse vorliegen, muss die Therapieentscheidung weiterhin anhand des individuellen (Risiko-)Profils des Patienten im interdisziplinären Konsens getroffen werden.

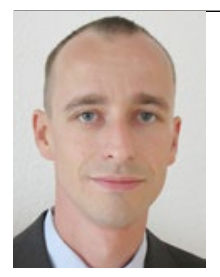

Dr. med. Sven Lerch Abteilung Neurologie, Bundeswehrkrankenhaus Berlin 